\title{
Isolation and Characterization of Novel Chlorpyrifos Degrading Fungus Isaria Farinosa
}

\author{
Kolengaden Paulson Karolin ${ }^{1 *}$, Kumarannair Saradadevi Meenakumari ${ }^{2}$ and Padmakumari Subha ${ }^{3}$ \\ 1. Post Graduate Student Department of Agricultural Microbiology, Kerala Agricultural University, College of Agriculture, \\ Vellayani 695 522, Kerala, India \\ 2. Professor Department of Agricultural Microbiology, Kerala Agricultural University, College of Agriculture, Vellayani 695 522, \\ Kerala, India \\ 3. Research Associate Department of Agricultural Microbiology, Kerala Agricultural University, College of Agriculture, Vellayani \\ 695 522, Kerala, India
}

\begin{abstract}
Chlorpyrifos is a well known organophosphorus pesticide used worldwide. Microorganisms including bacteria, fungi and actinomycetes have been reported to be efficient degraders of chlorpyrifos. The present study was successful in isolating a novel fungus that could degrade chlorpyrifos effectively upto 800 ppm concentration. Morphological and molecular characterization studies revealed the identity of the fungus as Isaria farinosa.
\end{abstract}

Key words: Chlorpyrifos, bioremediation, fungus, concentrations, intervals, degradation.

\section{Introduction}

Chlorpyrifos is one of the dominated broad spectrum organophosphorus insecticides inhibiting the neuron function of sucking, chewing and boring insects both in crop and soil [1]. It is reported to have a moderately high persistence in soil. The wide use of these pesticides especially chlorpyrifos over the years have resulted in environmental pollution, human and animal health hazards [2]. Considering the inherent toxic nature of these pesticides it is essential to remove them from the environment employing suitable remedial measures. Bioremediation exploiting microbial technology is one of the recent techniques for environmental clean-up. In the process, heterotrophic microorganisms break down hazardous compounds to obtain carbon and energy [3]. Generally bacteria are reported to be efficient degraders of chlorpyrifos. The first bacteria, that could degrade organophoshorus compounds was isolated in 1973 [4]

\footnotetext{
*Corresponding author: Kolengaden Paulson Karolin, post graduate student, research field: agricultural microbiology. E-mail: karolin153@gmail.com.
}

and identified as Flavobacterium sp. Since then several bacteria and a few fungal species have been isolated which can degrade a wide range of organophoshorus compounds in liquid cultures and soil systems. There are also many reports of chlorpyrifos degradation by fungal species. Fungi like Trichoderma, Phanerochaete, Aspergillus, Penicillium [5], Phanerochaete chrysosporium [6] Hypholoma fasciculare and Coriolus versicolor [7] are some of the examples of fungal degraders of chlorpyrifos. The present study could identify the fungi Isaria farinosa as an efficient degrader of the pesticide chlorpyrifos.

\section{Experimental Sections}

Soil samples were collected from cardamom plantations of Idukki district where chlorpyrifos drenching was regularly practiced by the farmers. Microorganisms capable of degrading chlorpyrifos were isolated from these samples by enrichment culture technique. For the purpose soil samples collected was enriched continuously with 100 ppm 
chlorpyrifos for a period of 8 to 10 weeks. After enrichment the soil was mixed uniformly and one gram of enriched soil was added to mineral salts medium [3, 8], supplemented with 100 ppm chlorpyrifos and incubated at room temperature. After one week, $1 \mathrm{~mL}$ from the above medium was transferred to fresh MS (mineral salts) medium containing chlorpyrifos and this was repeated 2-3 times for further purification. Finally $1 \mathrm{~mL}$ was plated on MS media containing 100 ppm chlorpyrifos. A fungal isolate (M5) was obtained which was identified in the later part of the study as Isaria farinosa. The growth of the isolate (M5) and degradation of chlorpyriphos in different concentrations of chlorpyrifos-100, 200, 400 and 800 ppm was evaluated. Significant growth and degradation of the isolate was recorded. An important observation was that mycelial mat production was significantly reduced but the isolate could produce significant colony count in MSM (mineral salts medium) medium.

\section{Results and Discussion}

\subsection{Results}

On 7th day of inoculation, in 100 ppm chlorpyrifos Isaria farinosa recorded a population build up of $72.50 \times 10^{3} \mathrm{cfu} \cdot \mathrm{mL}^{-1}$ and this increased to $100 \times 10^{3}$ $\mathrm{cfu} \cdot \mathrm{mL}^{-1}$ and $112.50 \times 10^{3} \mathrm{cfu} \cdot \mathrm{mL}^{-1}$ after 15 and 20 days after inoculation, respectively. Later the population slightly decreased to $110 \times 10^{3} \mathrm{cfu} \cdot \mathrm{mL}^{-1}$ and $72.50 \times 10^{3} \mathrm{cfu} \cdot \mathrm{mL}^{-1}$ during 25th and 30th day after inoculation respectively.

In $200 \mathrm{ppm}$ concentration the fungus-Isaria farinosa showed a population build up of $112.50 \times 10^{3}$ $\mathrm{cfu} \cdot \mathrm{mL}^{-1}, 113.50 \times 10^{3} \mathrm{cfu} \cdot \mathrm{mL}^{-1}$ and $122.50 \times 10^{3}$ cfu $\cdot \mathrm{mL}^{-1}$ after 7,15 and 20 days after inoculation respectively. During the 25th and 30th day, Isaria farinosa showed slight decrease in population build up which recorded $119 \times 10^{3} \mathrm{cfu} \cdot \mathrm{mL}^{-1}$ and $70 \times 10^{3}$ $\mathrm{cfu} \cdot \mathrm{mL}^{-1}$, respectively.

On 7th day of inoculation, in 400 ppm chlorpyrifos,
Isaria farinosa recorded a population build up of $128.50 \times 10^{3} \mathrm{cfu} \cdot \mathrm{mL}^{-1}$ and this increased to $130 \times 10^{3}$ $\mathrm{cfu} \cdot \mathrm{mL}^{-1}$ and $146 \times 10^{3} \mathrm{cfu} \cdot \mathrm{mL}^{-1}$ on 15th and 20th day after inoculation, respectively. On 25th day, Isaria farinosa recorded a population of $144 \times 10^{3} \mathrm{cfu} \cdot \mathrm{mL}^{-1}$ and the population declined to $77.50 \times 10^{3} \mathrm{cfu} \cdot \mathrm{mL}^{-1}$ on 30th day after inoculation.

The population was comparatively less in $800 \mathrm{ppm}$ concentration of chlorpyrifos and it recorded $36 \times 10^{3}$ $\mathrm{cfu} \cdot \mathrm{mL}^{-1}, 41.50 \times 10^{3} \mathrm{cfu} \cdot \mathrm{mL}^{-1}, 50 \times 10^{3} \mathrm{cfu} \cdot \mathrm{mL}^{-1}$, $48.50 \times 10^{3} \mathrm{cfu} \cdot \mathrm{mL}^{-1}$ and $49.50 \times 10^{3} \mathrm{cfu} \cdot \mathrm{mL}^{-1}$ after 7 , 15, 20, 25 and 30 days after inoculation, respectively ( shown in Table 1).

The degradation potential of Isaria farinosa was assessed by analyzing the residue of chlorpyrifos in the medium in GC-MS (gas chromatography-mass spectrometry). After 7 days of incubation the isolate Isaria farinosa showed $53.7 \%$ degradation of 100 ppm chlorpyrifos and this increased to 59.7, 59.5, 62.4 and 64.2 percent on 15th, 20th, 25th and 30 days after inoculation respectively (Table 2). Similarly the isolate could degrade 200 ppm chlorpyrifos which recorded 34.3, 46.3, 58.4, 73.6 and 77.3 percent on 7, 15, 20, 25 and 30 day after inoculation, respectively. In 400 ppm chlorpyrifos Isaria farinosa showed 62.4, $62.9,62.8,63.5$ and 80.8 percent reduction of residue of chlorpyrifos on 7th, 15th, 20th, 25th and 30th days after inoculation respectively. The fungus was also efficient in degrading $800 \mathrm{ppm}$ concentration of chlorpyrifos and recorded 60.4, 61.2, 61.5, 61.3 and 61.0 percent reduction of chlorpyrifos under in vitro conditions (Table 2).

The fungal isolate was grown on plates containing mineral salts medium for 6 days for studying the colony morphology. The fungus has white flat cottony growth. The upper side of colony appeared pure white and lower side showed pale colour in MSM. Lemon shaped transparent conidia are another characteristic feature of this fungi.

Total genomic DNA was isolated and the 18 SrRNA gene was amplified by PCR (polymerase 
Table 1 The population buildup of Isaria farinosa in different concentrations of chlorpyrifos in MSM.

\begin{tabular}{lccccc}
\hline $\begin{array}{l}\text { Concentrations } \\
(\mathrm{ppm})\end{array}$ & \multicolumn{5}{c}{ Viable count $\left(\mathrm{cfu} \times 10^{3} \mathrm{cfu} \cdot \mathrm{mL}^{-1}\right)$} \\
\cline { 2 - 6 } & 7th DAI & 15th DAI & 20th DAI & 25th DAI & 30th DAI \\
\hline 100 & 72.50 & 100.0 & 112.50 & 110.00 & 72.50 \\
200 & 112.50 & 113.50 & 122.50 & 119.00 & 70.00 \\
400 & 128.50 & 130.00 & 146.00 & 144.00 & 77.50 \\
800 & 36.00 & 41.50 & 50.00 & 48.50 & 49.50 \\
\hline
\end{tabular}

DAI: days after inoculation.

Table 2 Degradation of chlorpyrifos in MSM by Isaria farinosa at different intervals.

\begin{tabular}{|c|c|c|c|c|c|c|c|c|c|c|}
\hline \multirow{2}{*}{$\begin{array}{l}\text { Concentrations } \\
\text { (ppm) }\end{array}$} & \multicolumn{2}{|c|}{ 7th DAI } & \multicolumn{2}{|c|}{ 15th DAI } & \multicolumn{2}{|c|}{ 20th DAI } & \multicolumn{2}{|c|}{ 25th DAI } & \multicolumn{2}{|c|}{ 30th DAI } \\
\hline & $\begin{array}{l}\begin{array}{l}\text { Residue } \\
\text { (ppm) }\end{array}\end{array}$ & $\begin{array}{l}\% \\
\text { Reduction }\end{array}$ & $\begin{array}{l}\text { Residue } \\
\text { (ppm) }\end{array}$ & $\begin{array}{l}\% \\
\text { Reduction }\end{array}$ & $\begin{array}{l}\text { Residue } \\
\text { (ppm) }\end{array}$ & $\begin{array}{l}\% \\
\text { Reduction }\end{array}$ & $\begin{array}{l}\text { Residue } \\
\text { (ppm) }\end{array}$ & $\begin{array}{l}\% \\
\text { Reduction }\end{array}$ & $\begin{array}{l}\text { Residue } \\
\text { (ppm) }\end{array}$ & $\begin{array}{l}\% \\
\text { Reduction }\end{array}$ \\
\hline 100 & 42.3 & 53.7 & 37.1 & 59.7 & 37.05 & 59.5 & 34.05 & 62.4 & 32.1 & 64.2 \\
\hline 200 & 130 & 34.3 & 104.0 & 46.3 & 79.35 & 58.4 & 50.20 & 73.6 & 43.0 & 77.3 \\
\hline 400 & 137.1 & 62.4 & 135.2 & 62.9 & 135.1 & 62.8 & 132.1 & 63.5 & 69.3 & 80.8 \\
\hline 800 & 293.1 & 60.4 & 287.3 & 61.2 & 284.3 & 61.5 & 282.3 & 61.3 & 279.0 & 61.0 \\
\hline
\end{tabular}

Table 3 Residue of chlorpyrifos (ppm) in control at different intervals.

\begin{tabular}{|c|c|c|c|c|c|}
\hline \multirow{2}{*}{$\begin{array}{l}\text { Concentrations } \\
\text { (ppm) }\end{array}$} & 7th DAI & 15th DAI & 20th DAI & 25th DAI & 30th DAI \\
\hline & $\begin{array}{l}\text { Residue of } \\
\text { chlorpyrifos (ppm) }\end{array}$ & $\begin{array}{l}\text { Residue of } \\
\text { chlorpyrifos (ppm) }\end{array}$ & $\begin{array}{l}\text { Residue of } \\
\text { chlorpyrifos (ppm) }\end{array}$ & $\begin{array}{l}\text { Residue of } \\
\text { chlorpyrifos (ppm) }\end{array}$ & $\begin{array}{l}\text { Residue of } \\
\text { chlorpyrifos (ppm) }\end{array}$ \\
\hline 100 & 93.000 & 92.400 & 91.700 & 90.800 & 90.100 \\
\hline 200 & 198.00 & 194.00 & 192.00 & 191.00 & 190.00 \\
\hline 400 & 365.00 & 364.00 & 363.00 & 362.00 & 361.00 \\
\hline 800 & 741.40 & 740.20 & 737.40 & 729.60 & 724.30 \\
\hline
\end{tabular}

chain reaction) with the universal primers NS1 (GTAGTCATATGCTTGTCTC) and NS4 (CTTCCGTCAATTCCTTTAAG).

Molecular characterization the fungi showed similarity towards Isaria farinose (Fig. 1). The amplification of genomic DNA of fungi showed the amplicons of size between $1 \mathrm{~KB}$ and $1.5 \mathrm{~KB}$ were observed. The rDNA regions of isolate were sequenced by using the Big-Dye Terminator v3.1 Cycle Sequencing Kit. The quality was checked using Sequence scanner software v1 (Applied Biosystems). Sequence alignment and editing of the obtained sequence were carried out using Geneious Pro v5.1. Sequencing of the isolate was done using universal primers of rDNA (18 SrRNA). The sequences obtained from isolate were as follows:

$$
>\text { M5-18S }
$$

TTATACAGCGAAACTGCGAATGGCTCATTA
TATAAGTTATCGTTTATTTGATAGTACCTTACT ACTTGGATAACCGTGGTAATTCTAGAGCTAAT ACATGCTGAAAATCCCGACTTCGGAAGGGAG GTATTTATTAGATTAAAAACCAATGCCCTCTG GGCTCCTTGGTGATTCATGATAACTGTTCGAA TCGCACGGCCTTGCGCCGGCGATGGTTCATTC AAATTTCTTCCCTATCAACTTTCGATGTTTGGG TATTGGCCAAACATGGTCGCAACGGGTAACG GAGGGTTAGGGCTCGACCCCGGAGAAGGAGC CTGAGAAACGGCTACTACATCCAAGGAAGGC AGCAGGCGCGCAAATTACCCAATCCCGATTC GGGGAGGTAGTGACAATAAATACTGATACAG GGCTCTTTTGGGTCTTGTAATTGGAATGAGTA CAATTTAAATCTCTTAACGAGGAACAATTGGA GGGCAAGTCTGGTGCCAGCAGCCGCGGTAAT TCCAGCTCCAATAGCGTATATTAAAGTTGTTG TGGTTAAAAAGCTCGTAGTTGAACCTTGGGCC TGGCTGGCCGGTCCGCCTCACCGCGTGTACTG 


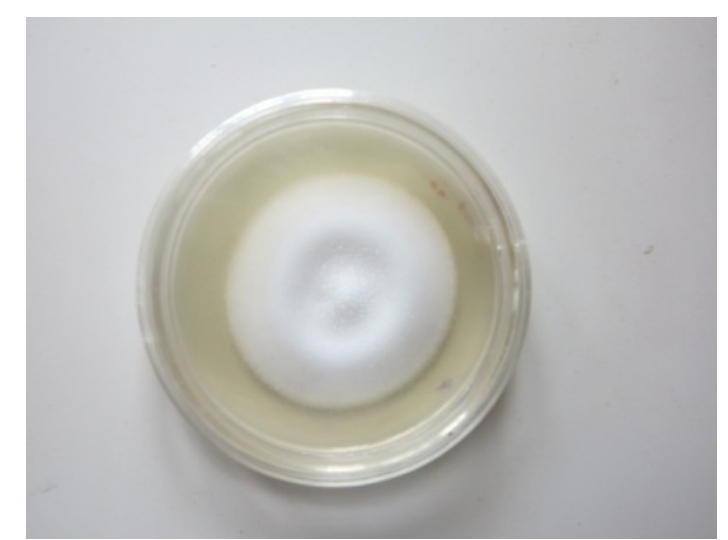

(a)

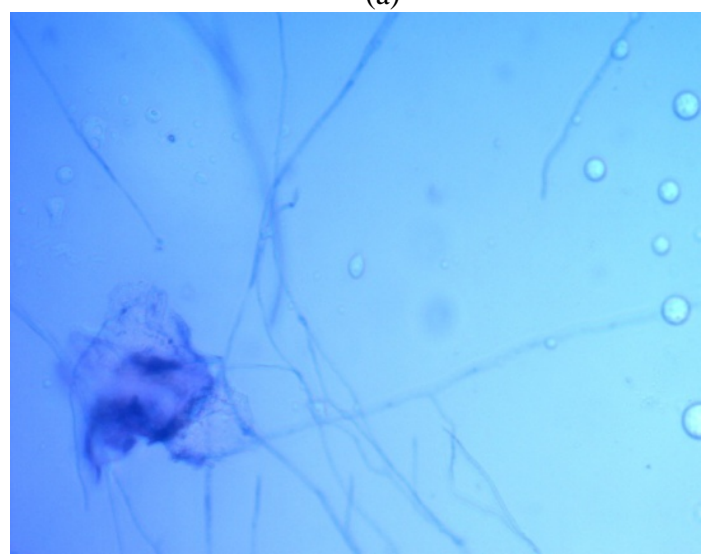

(b)

Fig. 1 Growth (a) and microscopic view (b) of Isaria farinose.

GTCCGGCCGGGCCTTTCCCTCTGTGGAACCTC ATGCCCTTCACTGGGTGTGGCGGGGAAACAG GACTTTTACTTTGAAAAAATTAGAGTGCTCCA GGCAGGCCTATGCTCGAATACATTAGCATGG AATAATAAAATAGGACGCGTGGTTCTATTTTG TTGGTTTCTAGGACCGCCGTAATGATTAATAG GGACAGTCGGGGGCATCAGTATTCAATTGTCA GAGGTGAAATTCTTAGATTTATTGAAGACTAA CTACTGCGAAAGCATTTGCCAAGGATGTTTTC ATTAATCAGGAACGAAAGTTAGGGGATCGAA GACGATCAGATACCGTCGTAGTCTTAACCATA AACTATGCCGACTAGGGATCGGACGATGTTAT TTTTTGACGCGTTCGGCACCTTACGAGAAATC AAAGTGCTT

The sequences obtained were analyzed using bioinformatics tool viz. BLASTN and tBLASTx i.e., nucleotide query is used to search in amino acid database. The fungi showed $100 \%$ similarity towards
Isaria farinosa with 1,023 bp and query sequence of 101 blast hits.

\subsection{Discussion}

An increase in growth with increase in concentration of chlorpyrifos was observed with Isaria farinosa. Even though the population buildup gradually increased with increase in concentration of chlorpyrifos up to $400 \mathrm{ppm}$, a decrease was observed at $800 \mathrm{ppm}$ concentration. The population buildup of Isaria farinosa was less in 800 ppm concentration compared to lower concentrations. The growth of Isaria farinosa was significantly reduced in $800 \mathrm{ppm}$ concentration of chlorpyrifos. A very high concentration usually leads to failure of biodegrdation because certain microorganisms are not resistant to such high concentrations. The significant number of active microbial population depends on resistant level of microbial strain and also the chemical nature of material to be degraded [9]. Another significant observation was that even though there was significant colony count; the mycelial mat formation was comparatively less in chlorpyrifos amended medium which is often advantageous for development of liquid formulations. Similar results were obtained by Bhalerao and Puranik [10], who noticed inhibition of mycelial growth of Aspergillus oryzae in flask with increasing concentration of chlorpyrifos. Similarly, mycelial mat formation was found to be reduced in Ganoderma sp. [11] and in Aspergillus terreus [12]. The isolate Isaria farinosa gave significant reduction of chlorpyrifos residue of 64.2 percent on 30 DAI in $100 \mathrm{ppm}$ concentration. A similar trend was also observed in 200, 400 and 800 ppm concentrations. Experiments conducted by Jones and Hastings [5], who could obtain 95 to 98 percent degradation of 50 ppm chlorpyrifos by a group of forest fungi namely Trichoderma harzianum, Penicillium vermiculatum and Mucor sp. after 28 days of incubation. Even though growth and degradation of chlorpyrifos by Isaria farinosa was comparatively less in 800 ppm 
concentrations about 60.4 percent degradation was observed on 7th day after inoculation and this trend continued even up to 30 days after inoculation which recorded 61.0 percent. In 800 ppm, maximum degradation was observed in 20-30 days. Hua [13] et al., also obtained similar results wherein maximum degradation of chlorpyrifos was observed on 35th day after inoculation. There are many earlier reports of other degrading chlorpyrifos but the degradation efficiency of Isaria farinosa has not been reported earlier.

\section{Conclusions}

The present study vividly established the efficiency of the novel fungi Isaria farinosa to degrade chlorpyrifos even up to 800 ppm concentration.

\section{Acknowledgments}

Authors are thankful to Rashtriya Krishi Vikas Yojana Project for the fund provided. Thanks are due to the faculty members of Department of Agricultural Microbiology, Entomology and Biotechnology for the technical support and guidance.

\section{References}

[1] Racke, K. D., Fontaine, D. D., Yoder, R. N., and Miller, J. R. 1994. "Chlorpyrifos Degradations in Soil at Termiticidal Application Rates.” Pesticide Sci. 42: 43-51.

[2] Sumit, K. 2011. "Isolation, Characterization and Growth Response Study of Chlorpyrifos Degrading Bacteria from Cultivated Soil.” Int. J. Adv. Eng. Technol. 2 (3): 199-203.

[3] Singh, B. K., Allan, S., Walker, A., Alun, J., Morgan, W., and Denis, J. 2004. "Biodegradation of Chlorpyrifos by Enterobacter Strain B-14 and Its Use in Bioremediation of Contaminated Soils.” Appl. Environ. Microbiol. 70:
4855-63.

[4] Dragun, J., Kuffner, A. C., and Schneiter, R. W. 1984. "Ground Water Contamination Transport and Transformation of Organic Chemicals.” Chem. Eng. 91: 65-70.

[5] Jones, A. S., and Hastings, F. L. 1981. "Soil Microbe Studies." In Field and laboratory evaluations of insecticides for southern pine beetle control, edited by Hastings F. L. and Coster, J. E. SE 21, USDA, Southern Forest Experiment Station, Forest Service, 13-14.

[6] Bumpus, J. A., Kakar, S. N., and Coleman, R. D. 1993. "Fungal Degradation of Organophosphorous Insecticides." Appl. Biochem. Biotechnol. 39 (40): 715-26.

[7] Bending, G. D., Friloux, M., and Walker, A. 2002. "Degradation of Contrasting Pesticides by White Rot Fungi and Its Relationship with Ligninolytic Potential.” FEMS Microbiol. Lett. 212: 59-63.

[8] Karpouzas, D. G., and Walker, A. 2000. "Factors Influencing the Ability of Pseudomonas Putida Strains ep I and II to Degrade the Organophosphate Ethoprophos.” $J$. Appl. Microbiol. 89: 40-8.

[9] Fang, H., Xiang, Y. Q., Hao, Y. J., Chu, X. Q., Pan, X. D., $\mathrm{Yu}, \quad$ J. Q. et al. 2008. "Fungal Degradation of Chlorpyrifos by Verticillium sp. DSP in Pure Cultures and its Use in Bioremediation of Contaminated Soil and Pakchoi." Int. Biodeterioration and Biodegradation 61: 294-303.

[10] Bhalerao, T. S., and Puranik, P. R. 2009. "Biodegradation of Organochlorine Pesticide, Endosulfan, by a Fungal Soil Isolate, Aspergillus Niger.” Int. Biodeterioration and Biodegradation 59: 315-21.

[11] Silambarasan, S., and Abraham, J. 2012. "Efficacy of Ganoderma sp. JAS4 in Bioremediation of Chlorpyrifos and its Hydrolyzing Metabolite TCP from Agricultural Soil.” J. Basic Microbiol. 54: 44-55.

[12] Silambarasan, S., and Abraham, J. 2013. "Ecofriendly Method for Bioremediation of Chlorpyrifos from Agricultural Soil by Novel Fungus Aspergillus Terreus JAS1.” Water Air Soil Pollut. 224: 1369.

[13] Hua, F., Yunlong, Y., Xiaoqiang, C., Xiuguo, W., Xiaoe, Y., and Jingquan, Y. 2009. "Degradation of Chlorpyrifos in Laboratory Soil and its Impact on Soil Microbial Functional Diversity.” J. Environ. Sci. 21: 380-6. 